\title{
Resonant Tunneling Diodes-Based Cellular Nonlinear Networks with Fault Tolerance Analysis
}

\author{
Shukai Duan, ${ }^{1}$ Xiaofang $\mathrm{Hu}^{2}{ }^{2}$ Lidan Wang, ${ }^{1}$ and Shiyong Gao ${ }^{1}$ \\ ${ }^{1}$ School of Electronics and Information Engineering, Southwest University, Chongqing 400715, China \\ ${ }^{2}$ Department of Mechanical and Biomedical Engineering, City University of Hong Kong, 83 Tat Chee Avenue, Kowloon, Hong Kong
}

Correspondence should be addressed to Lidan Wang; ldwang@swu.edu.cn

Received 31 January 2013; Accepted 9 May 2013

Academic Editor: Chuandong Li

Copyright (c) 2013 Shukai Duan et al. This is an open access article distributed under the Creative Commons Attribution License, which permits unrestricted use, distribution, and reproduction in any medium, provided the original work is properly cited.

\begin{abstract}
The resonant tunneling diodes (RTD) have found numerous applications in high-speed digital and analog circuits owing to its folded-back negative differential resistance (NDR) in current-voltage (I-V) characteristics and nanometer size. On account of the replacement of the state resistor in standard cell by an RTD, an RTD-based cellular neural/nonlinear network (RTD-CNN) can be obtained, in which the cell requires neither self-feedback nor a nonlinear output, thereby being more compact and versatile. This paper addresses the structure of RTD-CNN in detail and investigates its fault-tolerant properties in image processing taking horizontal line detection and edge extraction, for examples. A series of computer simulations demonstrates the promising faulttolerant abilities of the RTD-CNN.
\end{abstract}

\section{Introduction}

In 1988, Chua and Yang defined cellular neural/nonlinear network $(\mathrm{CNN})$ based on cellular automata and neural network $[1,2]$. CNN was acclaimed as a powerful back-end analog array processor and capable of accelerating various computation intensive tasks in image processing, motion detection, pattern formation and recognition, and robotics [3]. The structure of this network is easy to implement in very large integration (VLSI) technologies.

With the transistor size shrinking close to sub $100 \mathrm{~nm}$, many serious issues emerge, including the difficulty in fabrication and fundamental performance defects of transistor devices. It is believed that flash memory will approach the end of scaling down in a decade. Traditional transistor-based memory encounters the development bottleneck [4]. In order to overcome the limitations of CMOS, many nanometer-scale technologies have been proposed and utilized.

The devices and circuits based on quanta tunneling shows their advantages in nanometer-scale technology. Among several proposed nanometer electronic devices, the resonant tunneling diode possesses a relatively easy fabrication process and folded-back negative differential resistance (NDR) in current-voltage $(I-V)$ characteristics $[5,6]$, thereby exploring several applications in both digital and analog circuits [7]. Some researchers have also stated that RTD have extensive applications in Microwave oscillator circuit for terahertz and can be as the main prospect for the basic element of the new logic cell in high-speed digital integrated circuit.

By combining the advantages of RTD in NDR feature and $\mathrm{CNN}$ in parallel and high performance capacity in data processing, the hybrid RTD-CNN is expected to have higher speed, more compact structure, and greater capability [68]. However, the growing size in RTD-CNN increases the likelihood of faulting components existing in such neural networks. To some extent, we expect that RTD-CNNs with few faults may also provide acceptable results based on the inherent fault-tolerant property of artificial neural networks. It is also an essential task to evaluate the fault-tolerant ability of CNN. 
This paper investigates the resonant tunneling diodes based cellular neural/nonlinear networks (RTD-CNN) with fault-tolerant properties in image processing. In Section 2, the structure of the RTD-CNN is depicted in details, including the RTD model, CNN model, and the hybrid model. Then, in Section 3, fault tolerance, an important feature of RTD-CNNs, is analyzed, and the stability of RTD-CNNs with a single stuck-at fault is proved. A series of computer simulations and numerical analysis are given in Section 4. Conclusions are drawn in Section 5.

\section{Structure of the RTD-Based CNN Model}

2.1. Resonant Tunneling Diodes (RTDs). Nanometer electronics make it prospective of ultralow power and ultrahigh integration density. Among the different nanometer electronic devices discovered up to now, the resonant tunneling diode stands on the center of the stage. RTDs possess extreme compactness, picoseconds switching speed, nonmonotonic voltage-current characteristics, and possible monolithic and vertical integration with GaAs FETs [5].

The basic RTD device configuration, a double barrier quantum well structure, can be measured in nanometers (as Figure 1). The structure has two contacts (called the emitter and the collector) made from a semiconductor with a small bandgap (e.g., GaAs), quantum barriers made from a semiconductor with a larger bandgap (e.g., AlGaAs or InGaAs), and a quantum well made from a smaller bandgap semiconductor. The wave nature of the electrons in such a structure leads to quantum phenomena like interference, tunneling, and energy quantization. The quantum well is so narrow (about $5 \mathrm{~nm}$ ) that it can only contain a single energy level, the so-called resonant. Electrons wishing to travel from the emitter to the collector can only do so if they are lined up with this resonant energy level. The NDR property can be exploited to design compact bistable circuits without feedback in digital circuits.

The physical model of RTD has an effective mass approximation [8],

$$
\begin{aligned}
h(v)= & A \ln \left[\frac{1+e^{\left(B-C+n_{1} v\right) q / k T}}{1+e^{\left(B-C-n_{1} v\right) q / k T}}\right] \\
& \times\left[\frac{\pi}{2}+\arctan \left(\frac{C-n_{1} v}{D}\right)\right]+H\left(e^{n_{2} q v / k T}-1\right) .
\end{aligned}
$$

For the sake of simplicity, this characteristic can be approximated by a piecewise linear function as follows :

$$
\begin{aligned}
h(v)= & \alpha v+\beta\left(\left|v+v_{p}\right|-\left|v-v_{p}\right|\right) \\
& +\gamma\left(\left|v+v_{v}\right|-\left|v-v_{v}\right|\right) .
\end{aligned}
$$

The $I-V$ characteristics (NDR) of RTD is represented by a piece-wise linear model clearly. Initially, with a low voltage across the device (point A in Figure 2), the electrons are below

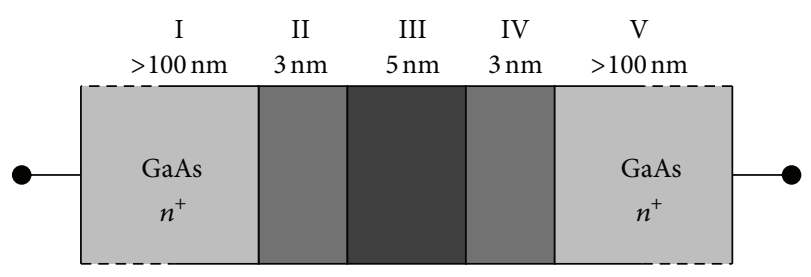

Quantum barriers, large bandgap (AIGaAs)

Quantum well, small bandgap (GaAs)

Figure 1: Resonant tunneling diode.

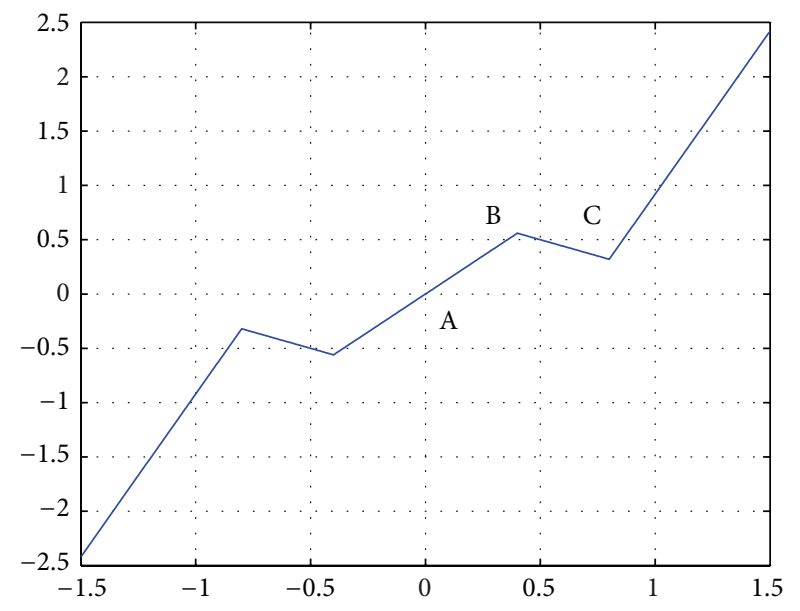

FIgURE 2: $I-V$ characteristics of the RTD.

the point of resonance, and no current can flow through the device. As the voltage increases, the emitter region is warped upwards, and the collector region is warped downwards. Eventually, the band of electrons in the emitter will line up with the resonant energy state and allows tunneling through to the collector (point B in Figure 2). With higher voltage, the electrons are pushed to pass the resonant energy level and are unable to continue tunneling, which can be observed by the drop in current to the valley at point $C$ in Figure 2. If the voltage increases further, more and more electrons are able to flow over the top of the quantum barriers, and the current flow will rise.

2.2. The Standard CNN. In recent twenty years, cellular neural networks have been greatly concerned and made significant progress in image processing and pattern recognition. The standard CNN circuit proposed by Chua and Yang is shown in Figure 3 [1]. The circuit of each cell in this network is shown in Figure 4, in which $C$ is a linear capacitor. $I_{x u}(i, j ; k, l)$ and $I_{x y}(i, j ; k, l)$ are linear voltagecontrolled current sources; $I_{y x}$ is a piecewise-linear voltagecontrolled current source; $R_{x}, R_{u}$, and $R_{y}$ are linear resistors; $E_{i j}$ is an independent voltage source. 


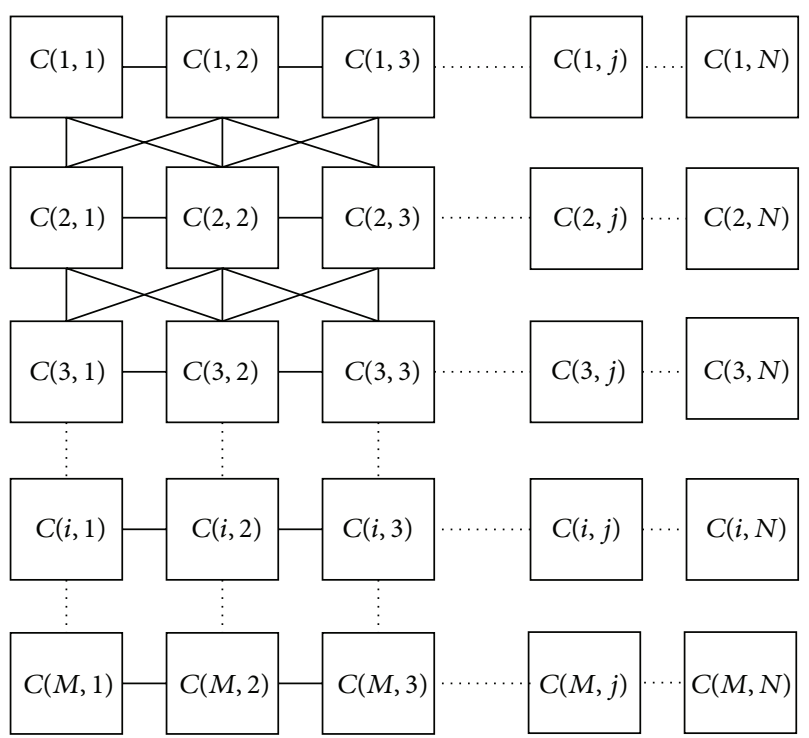

Figure 3: A $2 \mathrm{D} M \times N \mathrm{CNN}$.

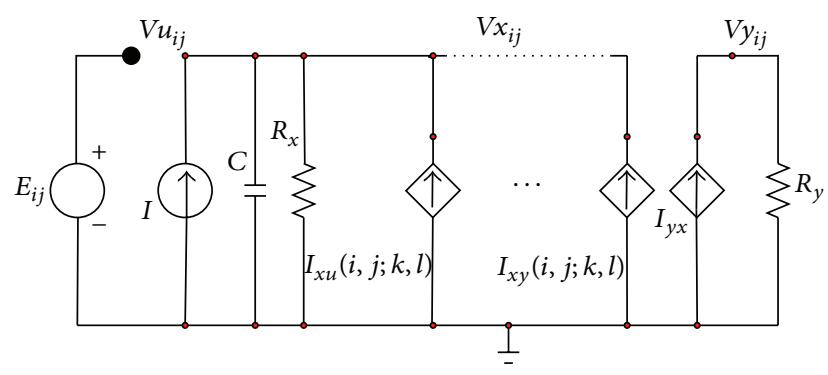

FIgURE 4: Schematic diagram of a CNN cell.

Applying KCL and KVL, the dynamics of a cell is derived as follows:

$$
\begin{aligned}
C \frac{d x_{i j}(t)}{d t}= & -\frac{1}{R} x_{i j}(t)+\sum_{C(k, l) \in N_{r}(i, j)} A(i, j ; k, l) f\left(x_{k l}(t)\right) \\
& +\sum_{C(k, l) \in N_{r}(i, j)} B(i, j ; k, l) u_{k l}(t)+I,
\end{aligned}
$$$$
1 \leq i \leq M ; 1 \leq j \leq N,
$$

$$
\begin{array}{r}
f\left(x_{i j}\right)=\frac{1}{2}\left(\left|x_{i j}(t)+1\right|-\left|x_{i j}(t)-1\right|\right), \\
1 \leq i \leq M ; 1 \leq j \leq N,
\end{array}
$$

where $N_{r}(i, j)$ is the $r$-neighborhood of a cell $(i, j)$ in a cellular neural network. $x_{i j}(t), u_{i j}$, and $f\left(x_{i j}\right)$ are the state, input and output of the cell, respectively.

\subsubsection{Remarks}

(a) All inner cells of a cellular neural network have the same circuit structures and parameter values. An

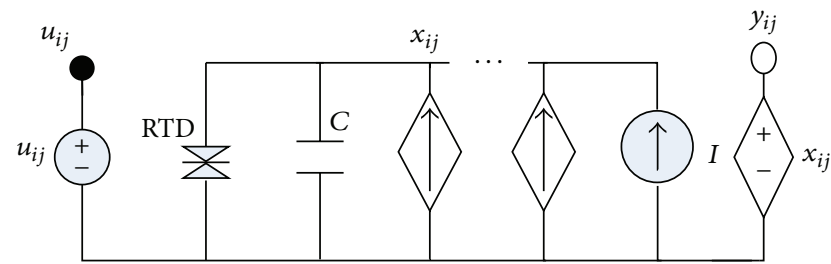

FIGURE 5: Schematic diagram of an RTD-CNN cell.

inner cell has $(2 r+1)^{2}$ neighbors. All other cells are called boundary cells whose neighborhood is usually set to 0 . A cellular neural network is completely characterized by the set of nonlinear differential equations (3a) and (3b).

(b) The dynamics of a cellular neural network has both output feedback and input control mechanisms. The output feedback effect depends on the interactive parameter $A(i, j ; k, l)$ and the input control effect depends on $B(i, j ; k, l)$. Consequently, it is sometimes instructive to refer to $A(i, j ; k, l)$ as a feedback operator and $B(i, j ; k, l)$ as a control operator.

2.3. The RTD-CNN Model. The linear resistor in the standard CNN cell is replaced by an RTD in an RTD-CNN cell (Figure 5). Because of this, the circuit becomes nonlinear, and the nonlinear relationship between state and output is unnecessary any longer. In this RTD-CNN cell, the state and output are identical; that is, $y_{i j}=x_{i j}$.

Let $h(\cdot)$ denotes the normalized piecewise linear characteristics of the RTD (as (2)). Considering the voltage across the RTD as the state $x$ of the cell, the dynamics of the RTD$\mathrm{CNN}$ cell is governed by

$$
C \frac{d x_{i j}(t)}{d t}=-h\left(x_{i j}(t)\right)+\sum_{(k, l)}\left(a_{i j, k l} x_{k l}(t)+b_{i j, k l} u_{k l}\right)+I .
$$

\section{The Stability Analysis of RTD-CNN with Single Faulty Cell}

Image processing is an important application for RTD-CNNs. It is always hoped that the network can still work smoothly even if there exists a faulty cell. For the sake of this purpose, the convergence feature of the RTD-CNN with single stuckat fault must be guaranteed. In this section, the stability of the proposed RTD-CNN has been analyzed and proved.

Definition 1. The stuck-at- $\alpha$ fault is defined that the state of a faulty cell is not changeable along with the inputs and outputs of other cells [9]:

$$
F=\alpha, \quad \alpha \text { is a constant. }
$$




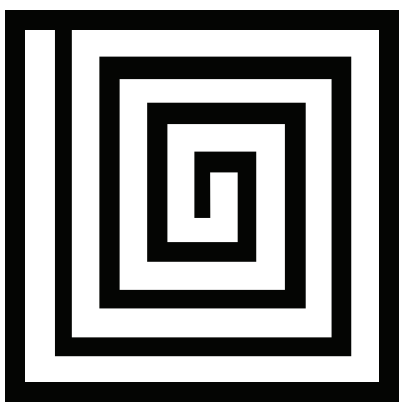

(a)

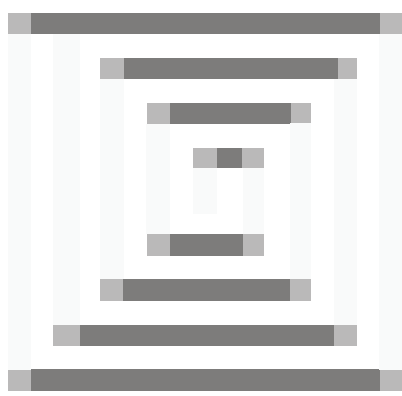

(b)

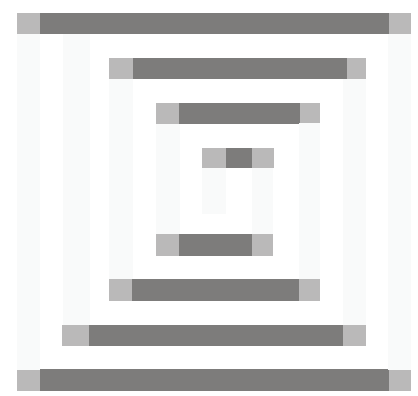

(c)

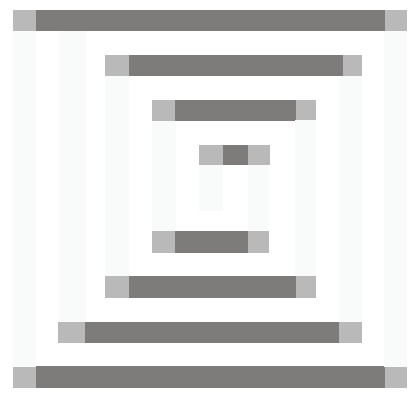

(d)

Figure 6: Horizontal line detection with RTD-CNN. (a) Input image, (b) output image, $t=20$, (c) output image, and $t=30$, (d) output image, $t=60$.

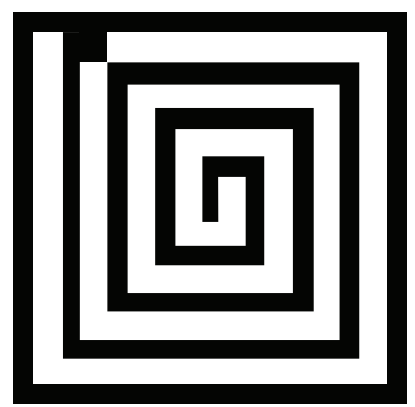

(a)

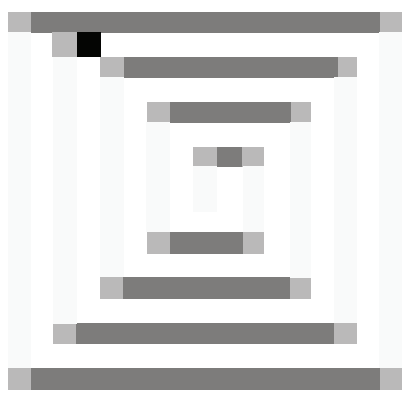

(b)

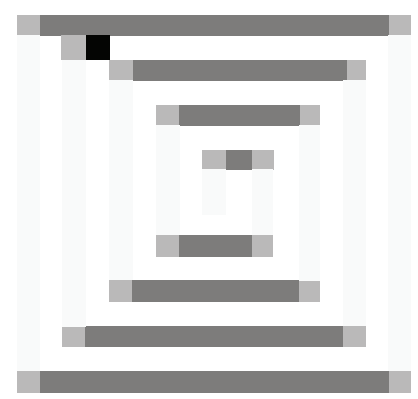

(c)

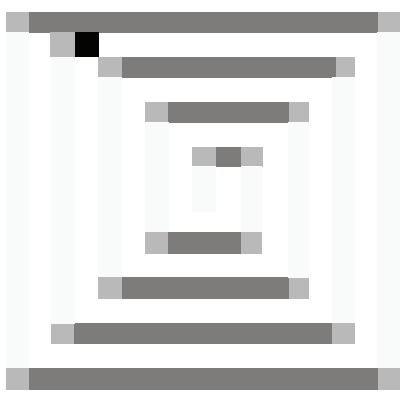

(d)

FIGURE 7: Horizontal line detection with RTD-CNN with single faulty cell. (a) Input image, (b) output image, $t=20$, (c) output image, $t=30$, (d) output image, $t=60$.

Then the dynamics of the RTD-CNN cell with single stuck-at fault is governed by

$$
\begin{aligned}
C \frac{d x_{i j}(t)}{d t}= & -h\left(x_{i j}(t)\right)+\sum_{(k, l)}\left(\bar{a}_{i j, k l} x_{k l}(t)+b_{i j, k l} u_{k l}\right) \\
& +I+\alpha_{f} \alpha,
\end{aligned}
$$

where $a_{f}$ and $\bar{a}$ are called the fault and rest template, respectively. Then, some main results can be given.

Definition 2. A Lyapunov function $E(t)$ for the RTD-CNN cell with single stuck-at- $\alpha$ fault is as follows:

$$
\begin{aligned}
E(t)= & -\frac{1}{2} \sum_{(i, j)} \sum_{(k, l)} \bar{a}_{i j, k l} x_{k l}(t) x_{i j}(t)-\sum_{(i, j)} \sum_{(k, l)} b_{i j, k l} u_{k l}(t) x_{i j}(t) \\
& -\sum_{(i, j)} I x_{i j}(t)+\sum_{(i, j)} \int_{0}^{x_{i j}} h(s) d s-\sum_{(i, j)} a_{f} \alpha x_{i j}(t) .
\end{aligned}
$$

Theorem 3. The function $E(t)$ is monotonically decreasing; that is,

$$
\frac{d E(t)}{d t} \leq 0
$$

Proof. If the feedback template is symmetric (i.e., $a_{i j, k l}=$ $a_{k l, i j}$ ), the derivative of the energy function with respect to time $t$ is

$$
\begin{aligned}
\frac{d E(t)}{d t}= & -\sum_{(i, j)} \sum_{(k, l)} \bar{a}_{i j, k l} x_{k l}(t) \frac{d x_{i j}(t)}{d t} \\
& -\sum_{(i, j)} \sum_{(k, l)} b_{i j, k l} u_{k l}(t) \frac{d x_{i j}(t)}{d t} \\
& -\sum_{(i, j)} I \frac{d x_{i j}(t)}{d t}+\sum_{(i, j)} \frac{d x_{i j}(t)}{d t} \frac{d}{d x_{i j}(t)} \\
& \times \int_{0}^{x_{i j}} h(s) d s-\sum_{(i, j)} a_{f} \alpha \frac{d x_{i j}(t)}{d t} .
\end{aligned}
$$

According to the definition of the cellular neural networks, we have

$a(i, j ; k, l)=0, \quad b(i, j ; k, l)=0, \quad$ for $C(k, l) \notin N_{r}(i, j)$. 


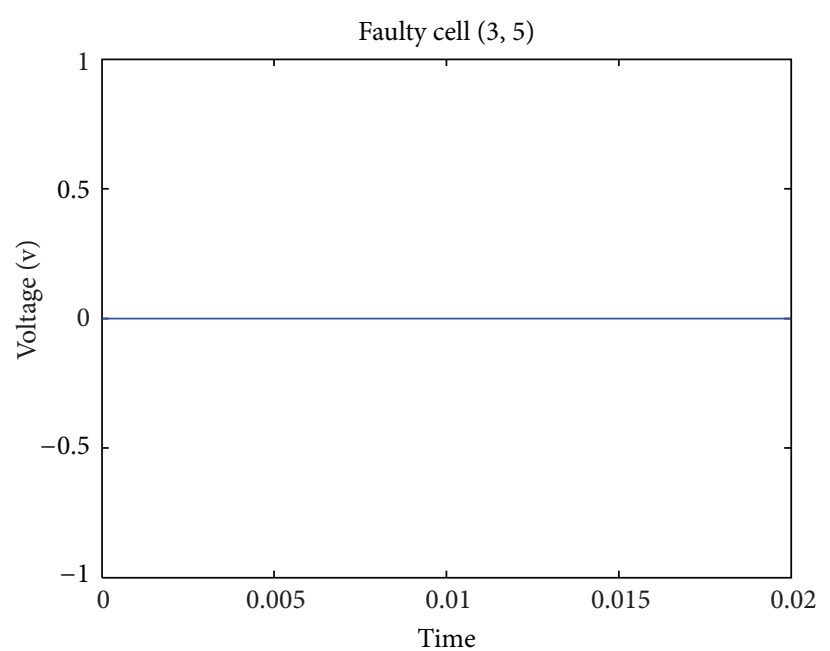

(a)

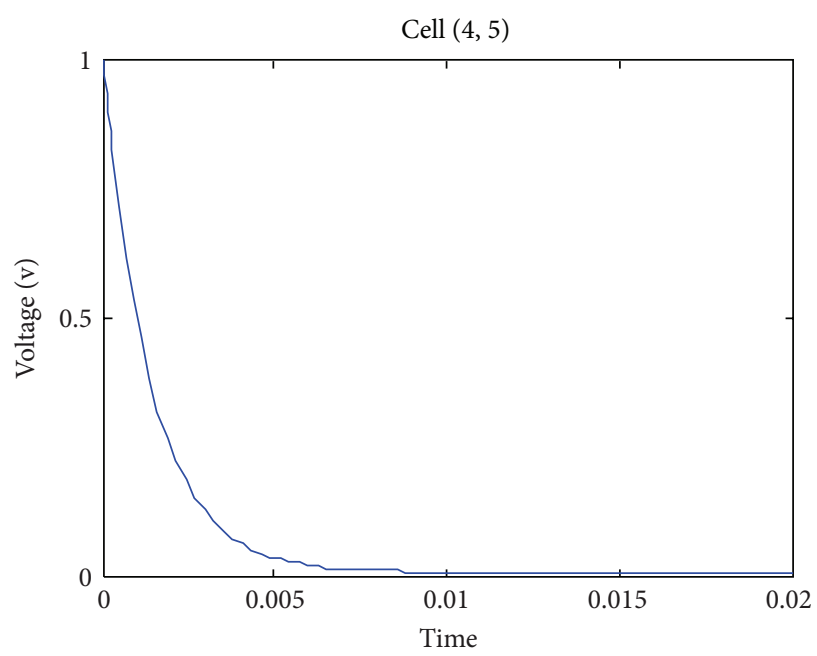

(c)

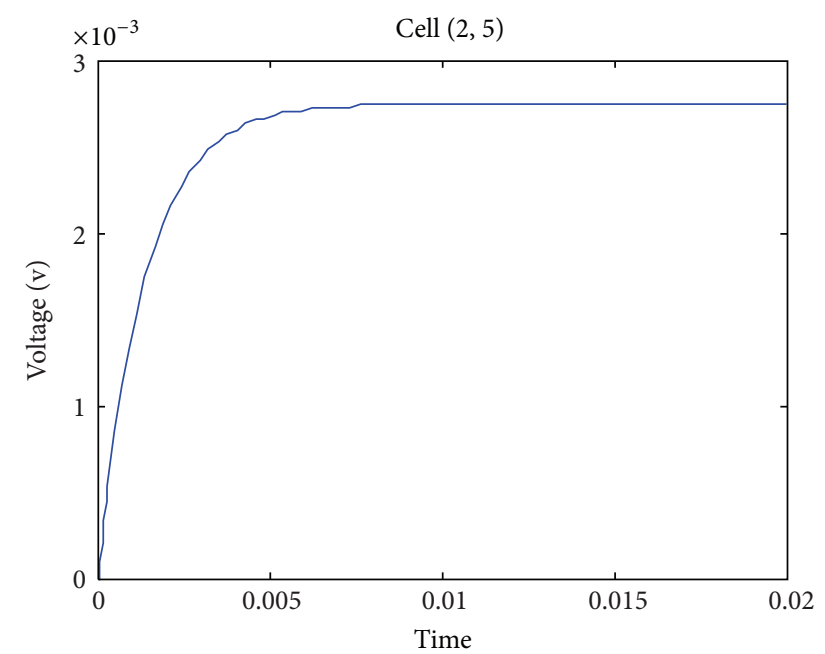

(b)

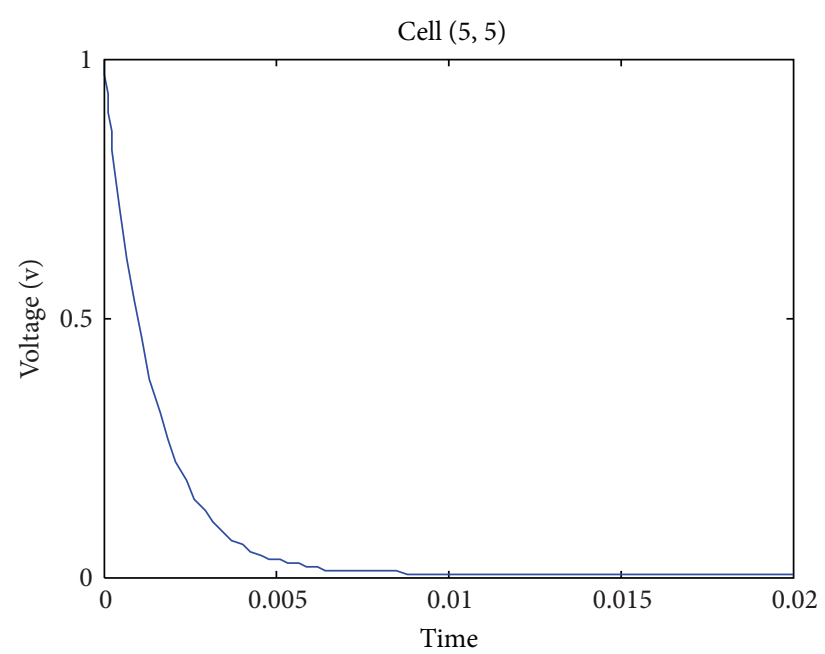

(d)

FIGURE 8: The dynamics of the state variable of cells in horizontal line detection of the RTD-CNN with single faulty cell.
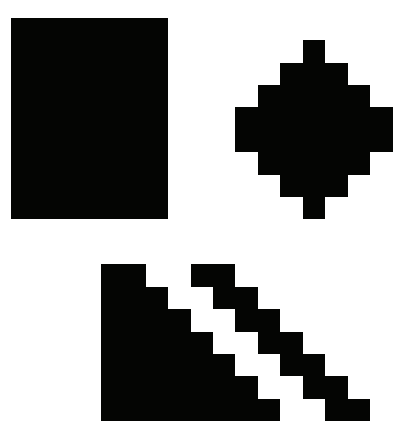

(a)

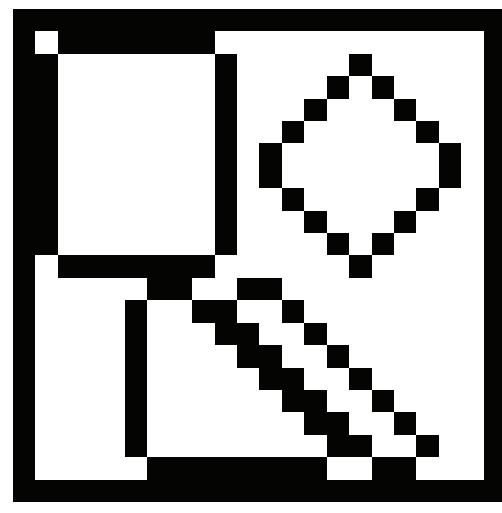

(b)

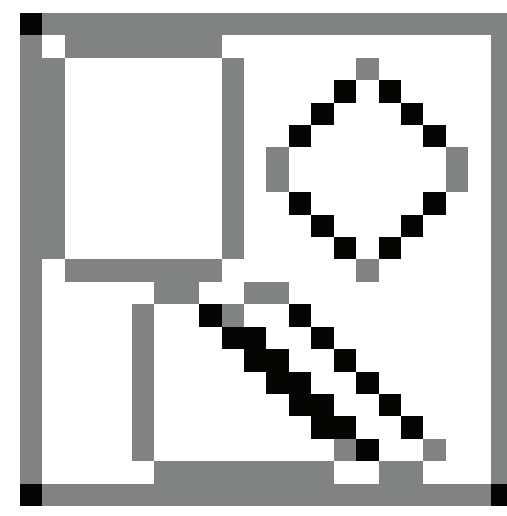

(c)

FIgURE 9: Edge extraction with RTD-CNN. (a) Input image, (b) output image, $q=1$, and (c) output image, $q=0.16$. 


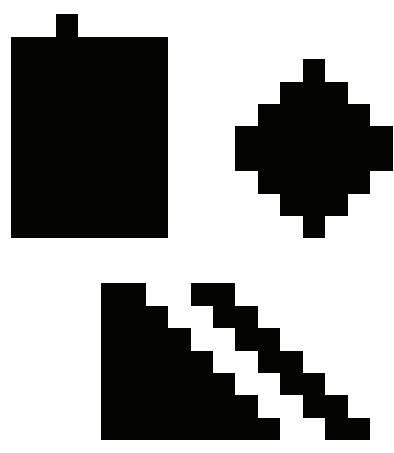

(a)

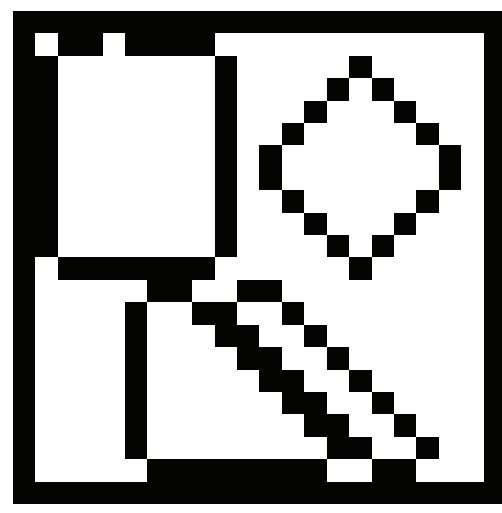

(b)

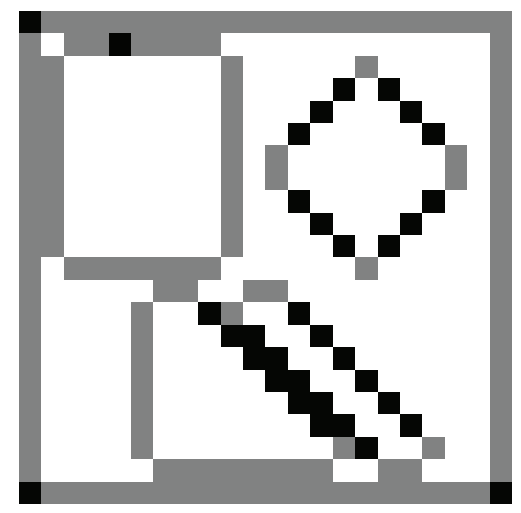

(c)

FIGURE 10: Edge extraction with RTD-CNN with a single faulty cell. (a) Input image, (b) output image, $q=1$, and (c) output image, $q=0.16$.

Then,

$$
\begin{aligned}
\frac{d E(t)}{d t}=-\sum_{(i, j)} \frac{d x_{i j}(t)}{d t}\left\{\sum_{(k, l)} \bar{a}_{i j, k l} x_{k l}(t)+\sum_{(k, l)} b_{i j, k l} u_{k l}(t)\right. \\
\left.+I-\frac{d}{d x_{i j}(t)} \int_{0}^{x_{i j}} h(s) d s+a_{f} \alpha\right\} \\
=-\sum_{(i, j)} \frac{d x_{i j}(t)}{d t}\left\{-h\left(x_{i j}(t)\right)\right. \\
+\sum_{(k, l)}\left(\bar{a}_{i j, k l} x_{k l}(t)+b_{i j, k l} u_{k l}(t)\right) \\
\left.+I+\alpha_{f} \alpha\right\} .
\end{aligned}
$$

Substitute (4) into (11)

$$
\frac{d E(t)}{d t}=-\sum_{(i, j)} C\left(\frac{d x_{i j}(t)}{d t}\right)^{2} \leq 0 .
$$

Obviously, from Theorem 3, it can be found that the proposed RTD-CNN with single faulty cell is stable, which is essential in image processing.

\section{Fault Tolerance in CNN}

Taking the inherent fault-tolerant property of the cell neural network into account, the RTD-CNN in the presence of faults may also provide acceptable effects. In this section, we investigate the fault-tolerant property of the RTD-CNN through two image processing examples.

4.1. Horizontal Line Detection with RTD-CNN. A $19 \times 19$ RTD-CNN is employed to implement the horizontal line detection task in image processing. The RTD-CNN template is given as:

$$
a=\left[\begin{array}{lll}
0 & 0 & 0
\end{array}\right], \quad b=\left[\begin{array}{lll}
1 & 2 & 1
\end{array}\right], \quad I=2 .
$$

Figures 6 and 7 show the outputs of the horizontal line detection of the RTD-CNN with the time evolvement, which illustrate that the RTD-CNN can detect the horizontal line (at time $t=0.02 \mathrm{~s}$ ) successfully. Figure 8 shows the dynamics of the state variable of cells in horizontal line detection of the RTD-CNN with single faulty cell, which can achieve steadiness in $0.01 \mathrm{~s}$. A RTD-CNN with single stuck-at- $\alpha$ faulty cell can also accomplish horizontal line detection task satisfactorily.

\subsection{Edge Extraction with RTD-CNN. A $22 \times 22 \mathrm{RTD}-\mathrm{CNN}$} is built to verify its edge extraction capacity in image processing. The RTD-CNN template is shown in the following equation:

$$
a=\left[\begin{array}{lll}
0 & 0 & 0 \\
0 & 0 & 0 \\
0 & 0 & 0
\end{array}\right], \quad b=\left[\begin{array}{ccc}
0 & -q & 0 \\
-q & 4 q & -q \\
0 & -q & 0
\end{array}\right], \quad I=0 .
$$

Figures 9 and 10 show the results of edge extraction of the RTD-CNN with different values of $q$. The RTDCNN discriminates between edge cells (black), cells around the edge (white), and "background" cells, whose steadiness state is 0 (grey) clearly. And corner cells can apparently be separated from other edge cells with a smaller value of $q$. It is evident that the RTD-CNN can extract the edge (at time $t=0.1 \mathrm{~s}$ ) successfully, in spite of the effect of a single faulty cell.

Figure 11 shows the dynamics of the state variable of cells in edge extraction of RTD-CNN with a single faulty cell which can achieve steadiness in $0.01 \mathrm{~s}$. Similarly, A RTD$\mathrm{CNN}$ with single stuck-at- $\alpha$ faulty cell can also accomplish edge extraction task satisfactorily.

\section{Conclusions}

This paper demonstrates the characteristics of the RTDs and addresses the RTD-based cellular neural/nonlinear network (RTD-CNN), which makes simple the CNN cell structure but maintains its promising capabilities. Next, the RTD-CNN with single stuck-at fault is implemented and its stability is 


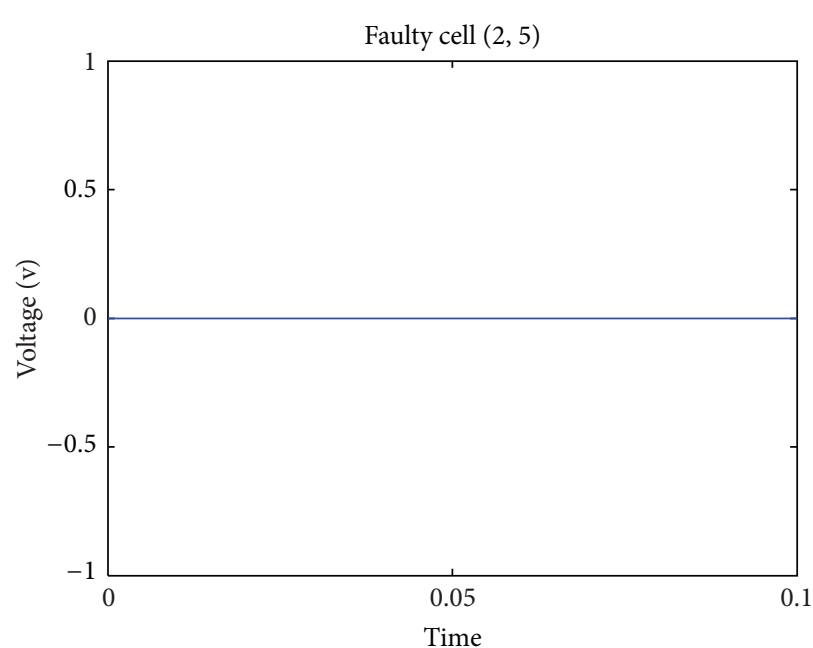

(a)

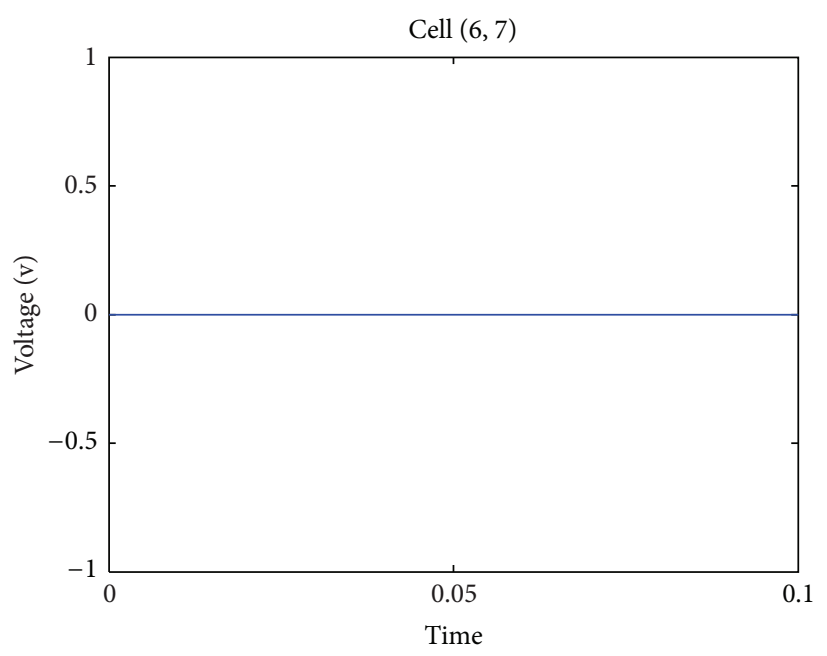

(c)

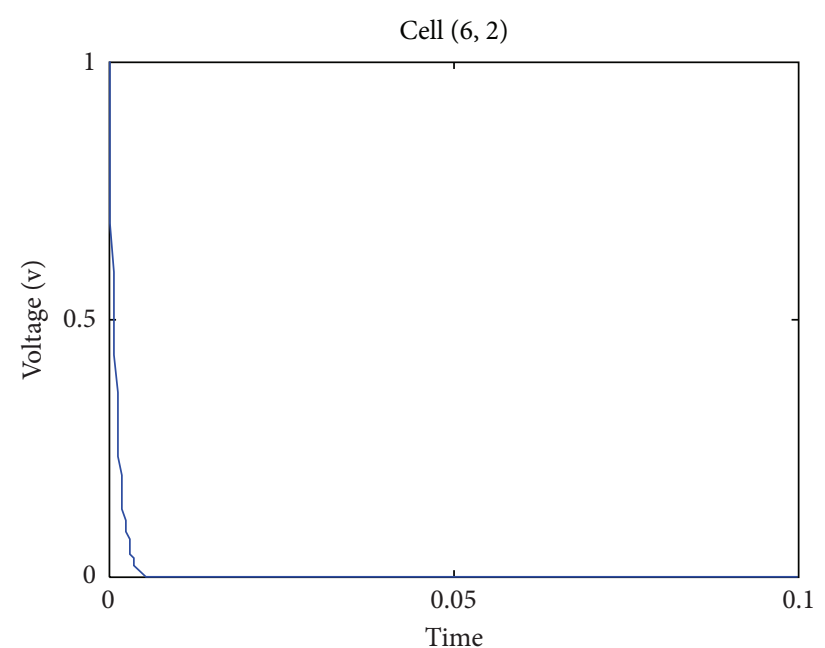

(b)

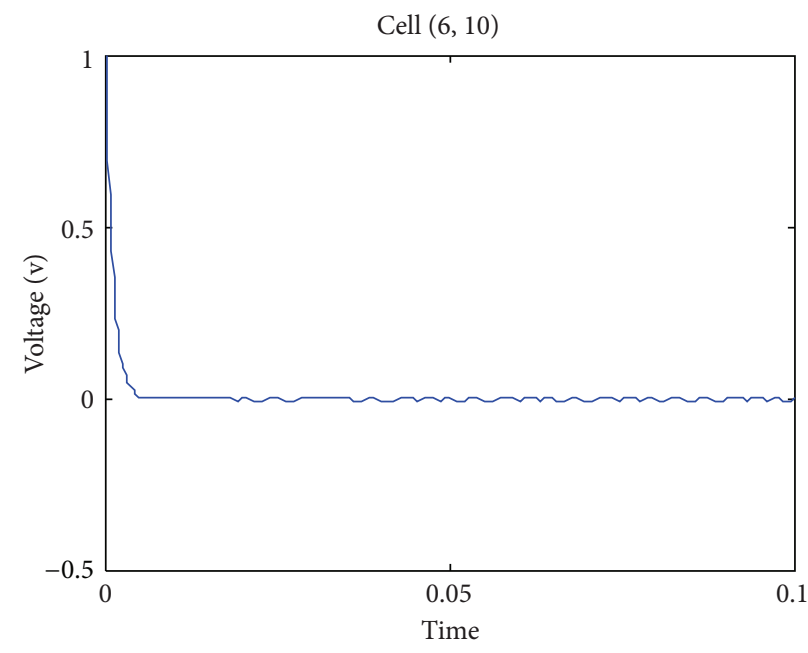

(d)

FIGURE 11: The dynamics of the state variable of cells in edge extraction of the RTD-CNN with a single faulty cell.

analyzed by Lyapunov stability theorem. Finally, the computer simulations of the RTD-CNN fully shows the satisfactory performance in image processing, such as horizontal line detection and edge extraction, improving the fault tolerance of RTD-CNN. Research results may provide a framework for further investigations in fault tolerant property of the RTDCNNs and its applications in image processing.

\section{Acknowledgments}

The work was supported by the National Natural Science Foundation of China (Grant Nos. 60972155 and 61101233), Fundamental Research Funds for the Central Universities (Grant Nos. XDJK2012A007 and XDJK2013B011), University Excellent Talents Supporting Foundations in of Chongqing (Grant No. 2011-65), University Key Teacher Supporting Foundations of Chongqing (Grant No. 2011-65), Technology Foundation for Selected Overseas Chinese Scholars, Ministry of Personnel in China (Grant No. 2012-186), and "Spring
Sunshine Plan" Research Project of Ministry of Education of China (Grant No. z2011148).

\section{References}

[1] L. O. Chua and L. Yang, "Cellular neural networks: theory," IEEE Transactions on Circuits and Systems, vol. 35, no. 10, pp. 1257$1272,1988$.

[2] L. O. Chua and T. Roska, Cellular Neural Networks and Visual Computing, Cambridge University Press, New York, NY, USA, 2002.

[3] L. O. Chua and T. Roska, "CNN paradigm," IEEE Transactions on Circuits and Systems I, vol. 40, no. 3, pp. 147-156, 1993.

[4] S. Duan, X. Hu, L. Wang, C. Li, and P. Mazumder, "Memristorbased RRAM with applications," Science China Information Sciences, vol. 55, no. 6, pp. 1446-1460, 2012.

[5] K. Karahaliloğlu and S. Balkir, "Nanostructure array of coupled RTDs as cellular neural networks," International Journal of Circuit Theory and Applications, vol. 31, no. 6, pp. 571-589, 2003. 
[6] M. Hnggi and L. O. Chua, "Cellular neural networks based on resonant tunnelling diodes," International Journal of Circuit Theory and Applications, vol. 29, no. 5, pp. 487-504, 2001.

[7] P. Mazumder, S. Kulkarni, M. Bhattacharya, J. P. Sun, and G. I. Haddad, "Digital circuit applications of resonant tunneling devices," Proceedings of the IEEE, vol. 86, no. 4, pp. 664-686, 1998.

[8] S. R. Li, P. Mazumder, and L. O. Chua, "Cellular neu$\mathrm{ral} /$ nonlinear networks using resonant tunneling diode," in Proceedings of the 4th IEEE Conference on Nanotechnology, pp. 164-167, IEEE, Munich, Germany, August 2004.

[9] L. D. Wang, X. F. Yang, and S. K. Duan, "Analysis of fault tolerance of cellular neural networks and applications to image processing," in Proceedings of the 3rd International Conference on Natural Computation, J. Lei, J. T. Yao, and Q. Zhang, Eds., pp. 252-256, IEEE Computer Society, 2007. 


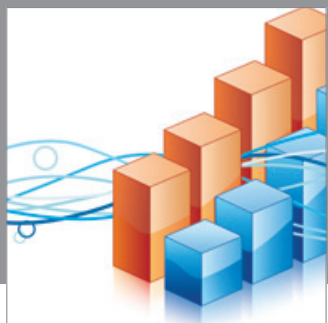

Advances in

Operations Research

mansans

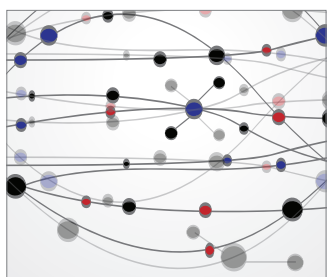

The Scientific World Journal
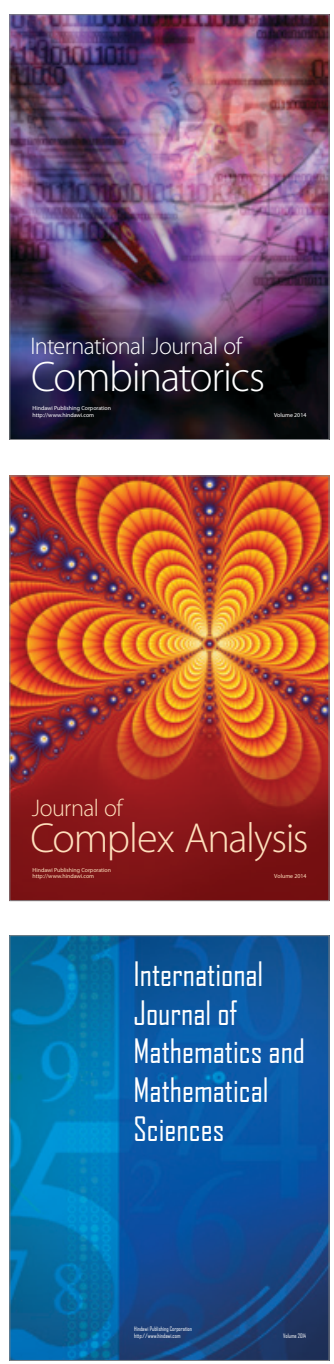
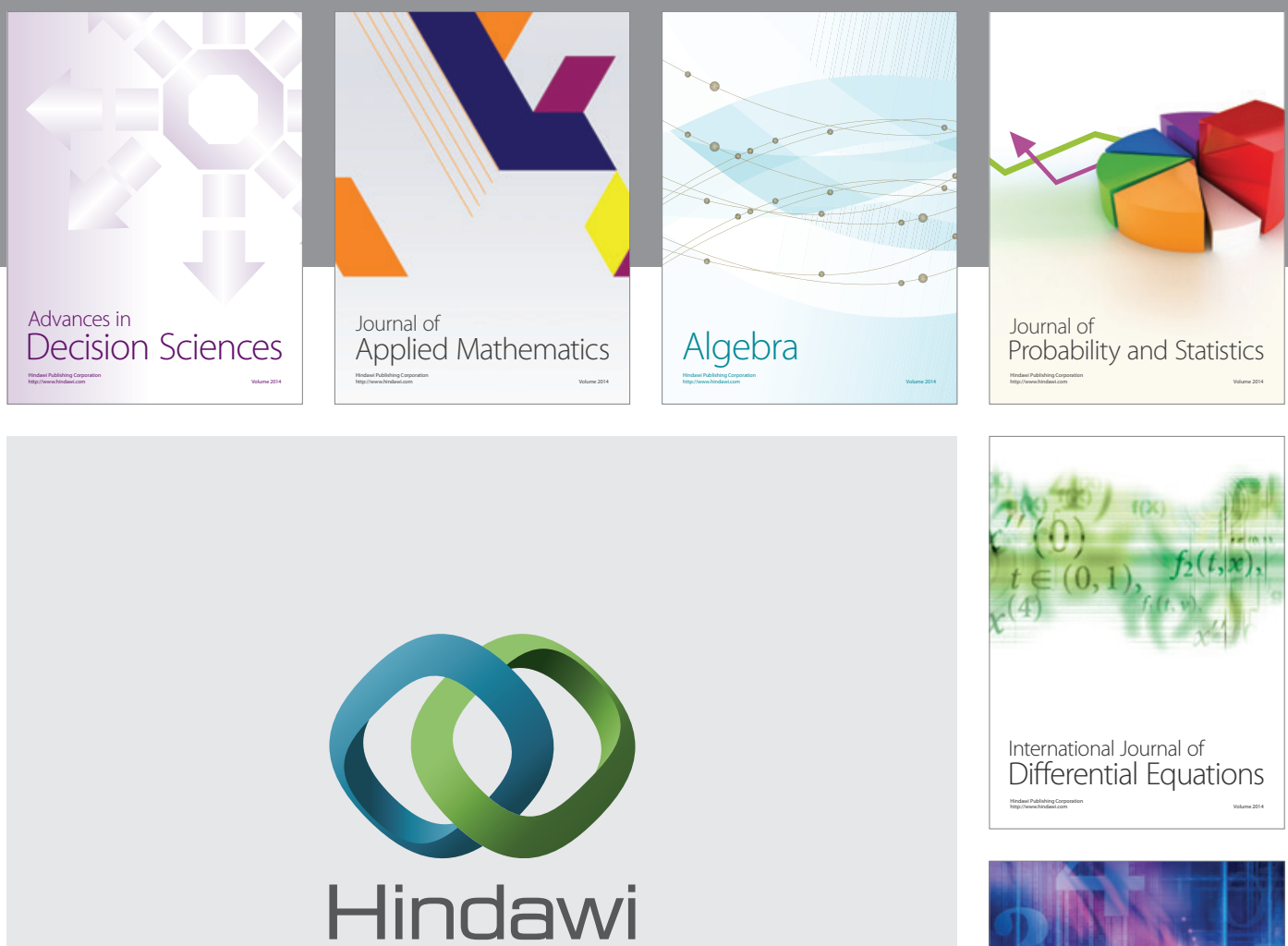

Submit your manuscripts at http://www.hindawi.com
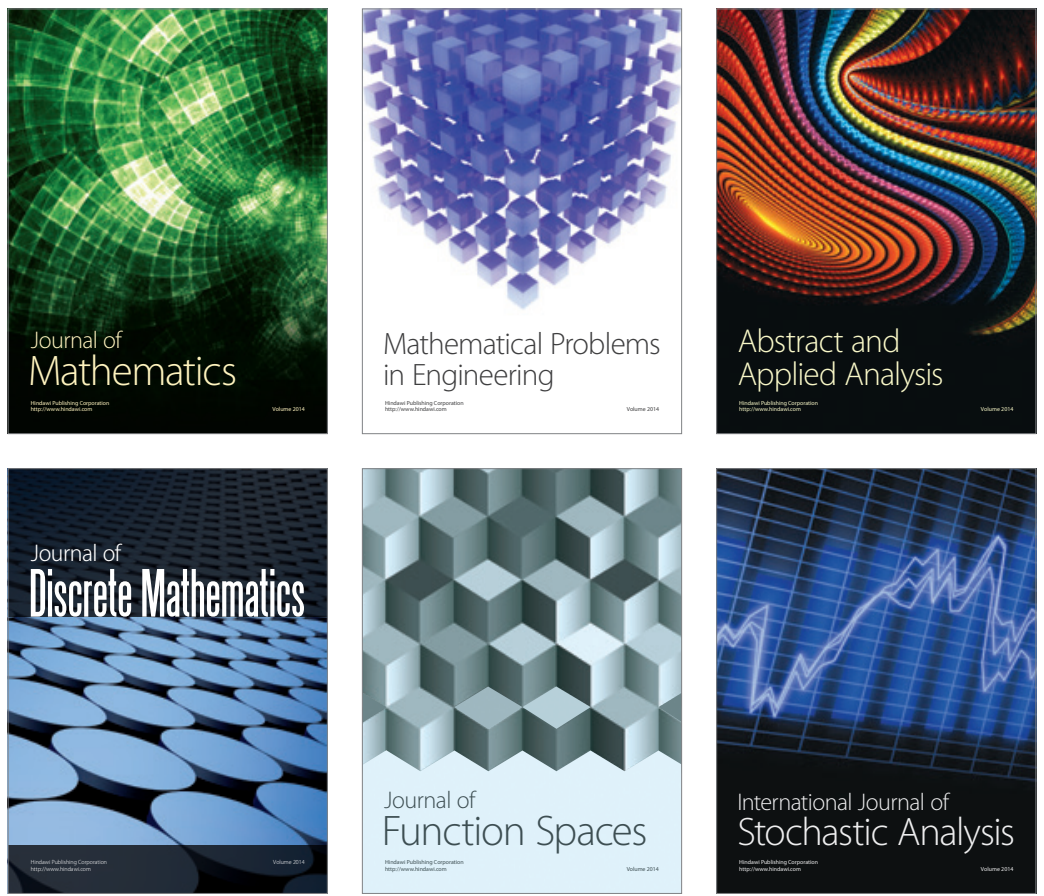

Journal of

Function Spaces

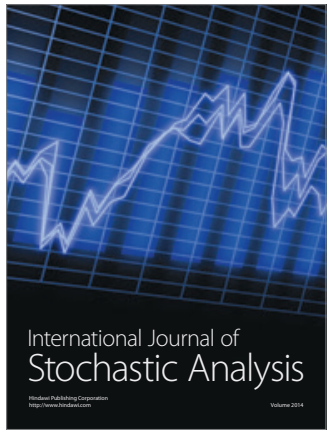

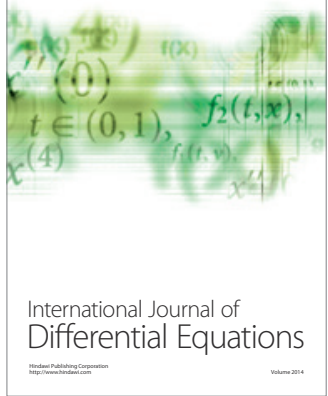
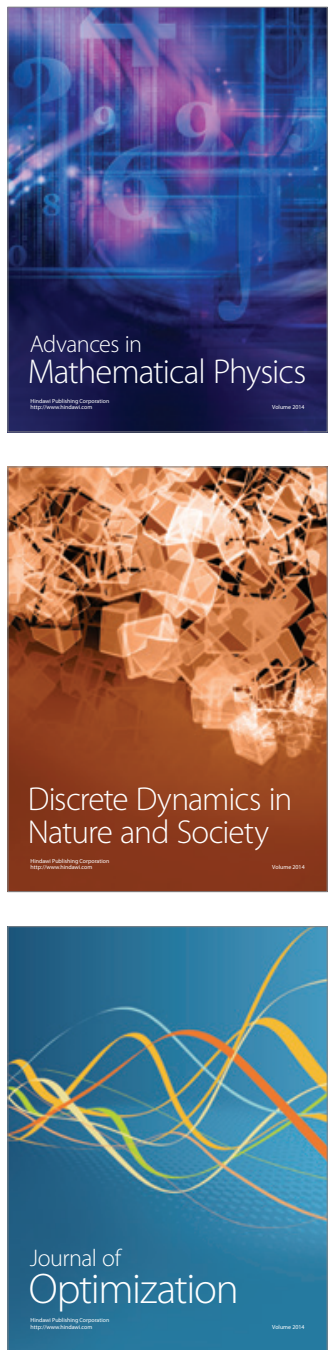